\title{
Reduced volumes of the CA1 and CA4-dentate gyrus hippocampal subfields in systemic lupus erythematosus
}

\author{
N Bódi ${ }^{1}$, A Polgár ${ }^{1}$, E Kiss ${ }^{1,2}$, Á Mester ${ }^{1}$, G Poór ${ }^{1,2}$ and S Kéri ${ }^{3,4,5}$ \\ ${ }^{1}$ National Institute of Rheumatology and Physiotherapy, Budapest, Hungary; ${ }^{2}$ Rheumatology Division of Third Department of Medicine, \\ Semmelweis University, Budapest, Hungary; ${ }^{3}$ Budapest University of Technology and Economics, Department of Cognitive Science, Budapest, \\ Hungary; ${ }^{4}$ Nyírő Gyula Hospital-National Institute of Psychiatry and Addictions, Budapest, Hungary; and ${ }^{5}$ University of Szeged, Department of \\ Physiology, Szeged, Hungary
}

\begin{abstract}
Introduction: There is evidence for hippocampal dysfunctions in systemic lupus erythematosus (SLE), which may contribute to neuropsychiatric impairments. However, fine structural alterations of the hippocampus have not been investigated in SLE. Methods: We measured the volume of hippocampal subfields in 18 SLE patients and 20 healthy control individuals matched for age, gender, and education. The MRI protocol included structural T1 volumes (Philips Achieva 3T scanner, magnetization-prepared rapid acquisition gradient echo (MPRAGE)). For image processing, we used the neuGRID platform and the longitudinal pipeline of FreeSurfer v6.0 with the "hipposubfields" flag. Results: Patients with SLE showed reduced volumes of CA1 (Cornu Ammonis 1) and CA4-dentate gyrus subfields relative to the control individuals. Smaller CA1 volumes were associated with worse performance on the Addenbrooke's Cognitive Examination. Conclusions: These preliminary results indicate a prominent vulnerability and functional relevance of the CA1 hippocampal subfield in SLE. Lupus (2017) 26, 1378-1382.
\end{abstract}

Key words: Hippocampus; magnetic resonance imaging; systemic lupus erythematosus; cognition

\section{Introduction}

Several studies reported neuropsychiatric manifestations in patients with systemic lupus erythematosus (SLE). The American College of Rheumatology (ACR) published a case definition and recommendations for neuropsychiatric SLE in which 19 different symptoms and syndromes were included. ${ }^{1}$ The prevalence of neuropsychiatric manifestations in SLE patients may be as high as $80 \%$ when the ACR nomenclature is considered. Regarding cognitive dysfunctions, most studies suggest a cross-sectional frequency of around $20 \%$ in SLE even when no other prominent neuropsychiatric symptoms are observable. ${ }^{2-5}$

From a broad range of cognitive dysfunctions, memory impairment is often reported in patients with SLE, which is consistent with hippocampal

Correspondence to: S Kéri, Department of Cognitive Science, Budapest University of Technology and Economics, Egry str. 1, Budapest, H1111, Hungary.

Email: szkeri@cogsci.bme.hu

Received 10 January 2017; accepted 6 March 2017 changes associated with the disease., ${ }^{5,6}$ There is structural neuroimaging evidence for reduced volumes of the hippocampal formation, corpus callosum, cerebellum, cerebral cortex, and amygdala in SLE. ${ }^{7-12}$ Functional neuroimaging studies demonstrated altered metabolism and functional connectivity in the prefrontal cortex, inferior parietal cortex, hippocampus, and anterior cingulate cortex of patients with SLE. ${ }^{5,13,14}$

The hippocampal formation is not a homogeneous structure: It consists of several histologically distinguishable modules, such as Cornu Ammonis (CA) regions, dentate gyrus (DG), presubiculum, and subiculum. These regions of the hippocampus are associated with different functions (e.g. memory encoding and retrieval) and may be specifically disrupted in various diseases. ${ }^{15,16}$ Despite the significance of this structural and functional specialization, to our knowledge, no studies have been conducted to elucidate possible subfield-specific hippocampal alterations in SLE. The aim of the present study was to examine the possibility that circumscribed subfields of the hippocampus show volumetric alterations in SLE. 


\section{Methods}

\section{Participants}

We recruited 18 female SLE patients who met the criteria of the ACR. ${ }^{1}$ There was no history of neuropsychiatric manifestations. The patients were compared with 20 healthy volunteers matched for age, education, and gender. Cognitive and neuropsychological assessment included the Mini-Mental State Examination (MMSE) ${ }^{17}$ and the Addenbrooke's Cognitive Assessment (ACE) ${ }^{18}$ The clinical state of the patients was characterized by the Systemic Lupus Disease Activity Index (SLEDAI) ${ }^{19}$ and the Systemic Lupus International Collaborating Clinic/ACR damage index (SLICC/ACR DI). ${ }^{20}$ All patients received steroid anti-inflammatory drugs (prednisolone, methylprednisolone), and six patients also received disease-modifying antirheumatoid drugs (methotrexate, leflunomide, and chloroquine). The clinical and demographic data are shown in Table 1.

All patients provided written informed consent and the study was conducted in accordance with the Declaration of Helsinki. The procedure was approved by the national ethics board (ETTTUKEB).

\section{Structural magnetic resonance imaging}

We followed the protocol of Marizzoni et al., ${ }^{21}$ which revealed longitudinal reproducibility of automated volumetric measurements of hippocampal subfields in several European centers. The protocol included structural T1 volumes (Philips Achieva 3T scanner, magnetization-prepared rapid acquisition

Table 1 Clinical and demographic characteristics

\begin{tabular}{lll}
\hline & $S L E(\mathrm{n}=18)$ & $\begin{array}{l}\text { Control individuals } \\
(\mathrm{n}=20)\end{array}$ \\
\hline Age (years) & $43.4(12.0)$ & $45.0(11.9)$ \\
Education (years) & $15.5(2.7)$ & $14.9(3.7)$ \\
MMSE & $29.3(1.2)$ & $29.8(0.6)$ \\
ACE & $91.7(4.4)$ & $95.4(4.1)$ \\
Age at disease onset (years) & $38.1(12.6)$ & - \\
SLEDAI & $5.2(2.6)$ & - \\
SLICC/ACR DI & $0.9(1.2)$ & - \\
Prednisolone equivalent & $8.3(5.4)$ & \\
$\quad$ corticosteroid dose & & \\
\hline
\end{tabular}

Data are mean (standard deviation). SLE: systemic lupus erythematosus; MMSE: Mini-Mental State Examination; ACE: Addenbrooke's Cognitive Assessment; SLEDAI: Systemic Lupus Disease Activity Index; SLICC/ACR DI: Systemic Lupus International Collaborating Clinic American College of Rheumatology damage index.

${ }^{a}$ Significant difference between SLE patients and control individuals, $t(36)=-2.62, p=0.01$. gradient echo (MPRAGE), three-dimensional sagittal acquisition, field of view $=240 \mathrm{~mm}$, $240 \times 256$ matrix, inversion time $=900 \mathrm{~ms}$, echo time $($ shortest $)=3.6 \mathrm{~ms}$, flip angle: 8 degrees, no fat suppression, partial $k$ space, no averages, acquisition time: 4 minutes and 17 seconds, acceleration factor: 1).

We used the neuGRID platform and the longitudinal pipeline of FreeSurfer v6.0 with the "hipposubfields" flag, which is a newer version of the software compared to that used by Marizzoni et $\mathrm{al}^{21}$ No manual adjustments were applied. We measured the volumes of CA1, CA2-CA3, CA4DG), subiculum, and presubiculum. This validated method is widely used in clinical research. ${ }^{21,22}$ The exploratory analyses did not show significant differences in left and right hippocampal subfields. Therefore, the data were averaged across hemispheres to reduce the number of variables in our small sample. Hippocampal subfield volumes were adjusted by using the total intracranial volume.

\section{Data analysis}

STATISTICA 12 (StatSoft, Tulsa, OK, USA) software was used for data analysis. SLE patients and control individuals were compared with two-tailed $t$-tests, corrected for multiple comparisons by using the false discovery rate (FDR) method. Effect size values (Cohen's $d$ ) were also calculated. Pearson's product moment correlation coefficients were calculated between hippocampal subfield volumes and cognitive measures. We used partial correlations to control for age, gender, and education. The level of statistical significance was set at alpha $<0.05$.

\section{Results}

Table 2 shows the hippocampal subfield volumes. There were significant volume reductions in SLE patients relative to control individuals in CA1 and CA4-DG subregions (Figure 1). The remaining hippocampal subfield did not show volumetric alterations in SLE.

SLE patients showed a mild but statistically significant cognitive impairment on the ACE scale (Table 1). There was a significant positive correlation between ACE scores and CA1 volumes in SLE (higher ACE scores reflecting better cognitive functions were associated with larger CA1 volumes) $(r=0.62, p=0.005)$. The remaining hippocampal subfields did not correlate with the ACE scores $(-0.2<r \mathrm{~s}<0.2)$. MMSE was not sensitive enough 
Table 2 Hippocampal subfield volumes $\left(\mathrm{mm}^{3}\right)$

\begin{tabular}{llllll}
\hline & SLE $(\mathrm{n}=18)$ & $\begin{array}{l}\text { Control individuals } \\
(\mathrm{n}=20)\end{array}$ & $\mathrm{t}$ & $\mathrm{p}$ & $\mathrm{d}$ \\
\hline CA1 & $281.6(71.4)$ & $339.1(56.6)$ & -2.76 & $0.009^{\mathrm{a}}$ & 0.9 \\
CA2-CA3 & $825.3(85.3)$ & $858.4(74.3)$ & -1.28 & 0.21 & 0.4 \\
CA4-DG & $483.6(85.9)$ & $577.7(72.7)$ & -3.66 & $0.0008^{\mathrm{a}}$ & 1.2 \\
Subiculum & $571.1(90.2)$ & $578.0(88.3)$ & -0.23 & 0.82 & 0.08 \\
Presubiculum & $398.8(91.9)$ & $408.5(74.6)$ & -0.54 & 0.59 & 0.1 \\
\hline
\end{tabular}

Data are mean (standard deviation). SLE: systemic lupus erythematosus; CA: Cornu Ammonis, DG: dentate gyrus.

${ }^{a}$ Significant differences after false discovery method corrections.

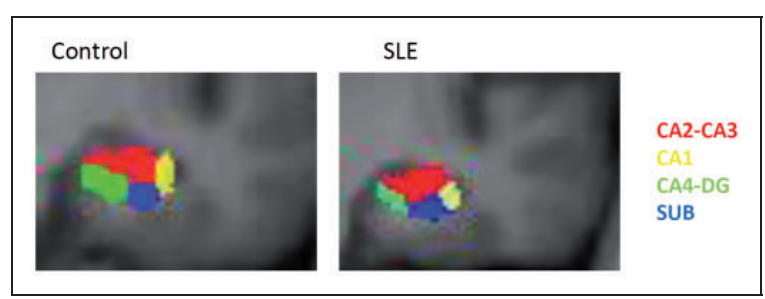

Figure 1 T1-weighted structural MRI images illustrating FreeSurfer hippocampal subfields in a healthy control individual and a patient with SLE. Atrophy of the CA1 and CA4-DG subfields is observable in SLE. MRI: magnetic resonance imaging; SLE: systemic lupus erythematosus; CA: Cornu Ammonis; DG: dentate gyrus; SUB: subiculum.

to detect between-group differences (Table 1), and its scores did not correlate with hippocampal volumes $(-0.2<r s<0.2)$. We found no significant correlations between hippocampal subfields, disease duration, clinical scales, and corticosteroid doses $(-0.1<r \mathrm{~s}<0.1)$.

\section{Discussion}

To the best of our knowledge, this is the first in vivo demonstration of selective hippocampal subfield deficits in SLE, namely, in the CA1 and CA4-DG areas. Interestingly, this deficit was observed in SLE patients with relatively preserved cognitive functions and no neuropsychiatric manifestations. Mild deficits on the ACE were associated with smaller CA1, but not CA4-DG, volumes.

There are a couple of previous studies that should be taken into consideration as elements of context of our results. In a patient who died from SLE with neuropsychiatric manifestations, Ballok et al. ${ }^{23}$ showed reduced neuronal density in CA3 and DG, but not in CA1, which is partially consistent with our in vivo findings from SLE patients without neuropsychiatric manifestations. In a longitudinal study including 150 patients with SLE, Appenzeller et al. ${ }^{7}$ identified hippocampal atrophy in $43.9 \%$ of patients, which increased to $66.7 \%$ by the end of the study. Hippocampal atrophy was associated with disease duration, total corticosteroid dose, history of central nervous system manifestations, and cognitive impairment. It is important to note that the circumscribed hippocampal volume loss described in our sample was not related to disease duration and corticosteroid dose, which can be explained by the small sample size. Recent results confirmed that hippocampal volume reduction is indeed frequently diagnosed in SLE, ${ }^{11}$ and the alteration of hippocampal signal intensity is a useful marker to determine patients with further progressive hippocampal atrophy. ${ }^{12}$ However, in SLE patients without neuropsychiatric manifestations not all studies reported hippocampal atrophy even though the patients showed a generalized cognitive deficit. ${ }^{24}$ A possible explanation is that less severely affected SLE patients are characterized by hippocampal subfield-specific volume reductions (CA1 and CA4-DG) as shown in the present study.

The CA1 subfield has a key role in learning and memory as the main output structure of the hippocampus. Most information leaves the hippocampus via CA1 and propagates to the neocortex. Within the hippocampus proper, CA1 is densely connected to DG via CA3. However, CA1 also receives direct pathways from the entorhinal cortex, which is a gateway to the hippocampus. Therefore, CA1 receives and compares two kinds of information: pre-processed and stored representations from the DG-CA3 system and new information from the entorhinal cortex. ${ }^{15,16}$ This mechanism may support the detection of a mismatch between internal representations and new environmental stimuli resulting in error signal processing (mismatch between expectations and actual sensory information) and novelty detection. ${ }^{25}$ On the other hand, recurrent and bidirectional DG-CA3 interactions may be linked to the sequential recall of memory events. $^{26}$ Interestingly, these hippocampal subregions display distinct electrophysiological properties, which may result in different functions in memory and spatial navigation. ${ }^{27}$

Bartsch et al. ${ }^{28}$ recently showed that the CA1 region is especially vulnerable to many types of metabolic and cytotoxic insults, including ischemia, inflammation (limbic encephalitis), status epilepticus, and transient global amnesia. Our results extend these findings, indicating that CA1 is also vulnerable in SLE and may specifically contribute to cognitive impairments. The possible mechanisms 
may include compromised synaptic plasticity, antibodies against the N-methyl-D-aspartate (NMDA) receptor, and decreased long-term potentiation and neurogenesis, which are candidate mechanisms for neuropsychiatric manifestations associated with chronic inflammatory conditions such as SLE. ${ }^{29,30}$ It is important to note that the patients in this study did not show severe neuropsychiatric symptoms, which indicates that structural abnormalities of the hippocampus are present in mild cases and contribute to subtle cognitive impairments.

Some limitations should be mentioned. First, the sample size was small and, therefore, the results are preliminary. Second, the cognitive assessment was limited to a few testing procedures that are not suitable for the evaluation of more specific cognitive functions. In addition, depression and fatigue must also be taken into consideration, although the current sample did not include SLE patients with major depressive or anxiety disorders. Future studies should investigate these issues.

In conclusion, SLE with subtle cognitive impairments is associated with reduced CA1 and DG-CA4 volumes, and CA1 atrophy is related to cognitive dysfunctions.

\section{Declaration of conflicting interests}

The authors declared no potential conflicts of interest with respect to the research, authorship, and/or publication of this article.

\section{Funding}

The authors received no financial support for the research, authorship, and/or publication of this article.

\section{References}

1 ACR Ad Hoc Committee on Neuropsychiatric Lupus Nomenclature. The American College of Rheumatology nomenclature and case definitions for neuropsychiatric lupus syndromes. Arthritis Rheum 1999; 42: 599-608.

2 Hanly JG, Fisk JD, Sherwood G, Jones E, Jones JV, Eastwood B. Cognitive impairment in patients with systemic lupus erythematosus. J Rheumatol 1992; 19: 562-567.

3 Hanly JG, Orowitz MB, Sanchez-Guerrero J, et al. Neuropsychiatric events at the time of diagnosis of systemic lupus erythematosus: An international inception cohort study. Arthritis Rheum 2007; 56: 265-273.

4 Brey RL, Holliday SL, Saklad AR, et al. Neuropsychiatric syndromes in lupus: Prevalence using standardized definitions. Neurology 2002; 58: 1214-1220.
5 Kozora E, Brown MS, Filley CM, et al. Memory impairment associated with neurometabolic abnormalities of the hippocampus in patients with non-neuropsychiatric lupus erythematosus. Lupus 2011; 20: 598-606

6 Csépány T, Bereczki D, Kollár J, Sikula J, Kiss E, Csiba L. MRI findings in central nervous system systemic lupus erythematosus are associated are associated with immunoserological parameters and hypertension. $J$ Neurol 2003; 250: 1348-1354.

7 Appenzeller S, Carnevalle AD, Li LM, Costallat LT, Cendes F. Hippocampal atrophy in systemic lupus erythematosus. Ann Rheum Dis 2006; 65: 1585-1589.

8 Appenzeller S, Bonilha L, Rio PA, Min Li L, Costallat LT, Cendes F. Longitudinal analysis of gray and white matter loss in patients with systemic lupus erythematosus. Neuroimage 2007; 34: 694-701.

9 Appenzeller S, Vasconcelos Faria A, Li LM, Costallat LT, Cendes F. Quantitative magnetic resonance imaging analyses and clinical significance of hyperintense white matter lesions in systemic lupus erythematosus patients. Ann Neurol 2008; 64: 635-643.

10 Muscal E, Traipe E, de Guzman MM, Myones BL, Brey RL, Hunter JV. Cerebral and cerebellar volume loss in children and adolescents with systemic lupus erythematosus: Review of clinically acquired brain magnetic resonance imaging. J Rheum 2010; 37: $1768-1775$.

11 Zimmermann N, Corrêa DG, Kubo TA, et al. Global cognitive impairment in systemic lupus erythematosus patients: A structural MRI study. Clin Neuroradiol 2017; 27: 23-29.

12 Lapa AT, Pedro T, Francischinelli J, et al. Abnormality in hippocampal signal intensity predicts atrophy in patients with systemic lupus erythematosus. Lupus. Epub ahead of print 22 November 2016.

13 Barraclough M, Elliott R, McKie S, Parker B, Bruce IN. Cognitive dysfunction and functional magnetic resonance imaging in systemic lupus erythematosus. Lupus 2015; 24: 1239-1247.

14 Mikdashi JA. Altered functional neuronal activity in neuropsychiatric lupus: A systematic review of the fMRI investigations. Semin Arthritis Rheum 2016; 45: 455-462.

15 Small SA, Schobel SA, Buxton RB, Witter MP, Barnes CA. A pathophysiological framework of hippocampal dysfunction in ageing and disease. Nat Rev Neurosci 2011; 12: 585-601.

16 Bartsch T, Wulff $P$. The hippocampus in aging and disease: From plasticity to vulnerability. Neuroscience 2015; 309: 1-16.

17 Folstein MF, Folstein SE, McHugh PR. Mini-Mental State. A practical method for grading the cognitive state of patients for the clinician. J Psychiatr Res 1975; 12: 189-198.

18 Dudas RB, Berrios GE, Hodges JR. The Addenbrooke's Cognitive Examination (ACE) in the differential diagnosis of early dementias versus affective disorder. Am J Geriatr Psychiatry 2005; 13: 218-226.

19 Bombardier C, Gladman DD, Urowitz MB, Caron D, Chang CH. Derivation of the SLEDAI. A disease activity index for lupus patients. The Committee on Prognosis Studies in SLE. Arthritis Rheum 1992; 35: 630-640.

20 Gladman D, Ginzler E, Goldsmith C, et al. The development and initial validation of the Systemic Lupus International Collaborating Clinics/American College of Rheumatology damage index for systemic lupus erythematosus. Arthritis Rheum 1996; 39: 363-369.

21 Marizzoni M, Antelmi L, Bosch B, et al. Longitudinal reproducibility of automatically segmented hippocampal subfields: A multisite European 3T study on healthy elderly. Hum Brain Mapp 2015; 36: $3516-3527$

22 Iglesias JE, Augustinack JC, Nguyen K, et al. A computational atlas of the hippocampal formation using ex vivo, ultra-high resolution MRI: Application to adaptive segmentation of in vivo MRI. Neuroimage 2015; 115: 117-137.

23 Ballok DA, Woulfe J, Sur M, Cyr M, Sakic B. Hippocampal damage in mouse and human forms of systemic autoimmune disease. Hippocampus 2004; 14: 649-661.

24 Kozora E, Arciniegas DB, Filley CM, et al. Cognition, MRS neurometabolites, and MRI volumetrics in non-neuropsychiatric systemic lupus erythematosus: Preliminary data. Cogn Behav Neurol 2005; 18: 159-162. 
Reduced volumes of the CA1 and CA4-dentate gyrus hippocampal subfields in SLE

$\mathrm{N}$ Bódi et al.

1382

25 Lisman JE, Otmakhova NA. Storage, recall, and novelty detection of sequences by the hippocampus: Elaborating on the SOCRATIC model to account for normal and aberrant effects of dopamine. Hippocampus 2001; 11: 551-568.

26 Lisman JE. Relating hippocampal circuitry to function: Recall of memory sequences by reciprocal dentate-CA3 interactions. Neuron 1999; 22: 233-242.

27 Oliva A, Fernández-Ruiz A, Buzsáki G, Berényi A. Spatial coding and physiological properties of hippocampal neurons in the Cornu Ammonis subregions. Hippocampus 2016; 26: 1593-1607.

28 Bartsch T, Döhring J, Reuter S, et al. Selective neuronal vulnerability of human hippocampal CAl neurons: Lesion evolution, temporal course, and pattern of hippocampal damage in diffusion-weighted MR imaging. J Cereb Blood Flow Metab 2015; 35 : 1836-1845.

29 Mackay M. Lupus brain fog: A biologic perspective on cognitive impairment, depression, and fatigue in systemic lupus erythematosus. Immunol Res 2015; 63: 26-37.

30 Jeltsch-David H, Muller S. Autoimmunity, neuroinflammation, pathogen load: A decisive crosstalk in neuropsychiatric SLE. J Autoimmun 2016; 74: 13-26. 